\title{
La biomasa de las lagunas costeras de la reserva de los Cayos Miskitos
}

\author{
The biomass of the coastal lagoons of the Miskito Cays Reserve
}

\author{
Donald Williams Smith ${ }^{1}$ \\ Delrin Chavarría Smith² \\ Marcos Williamson ${ }^{3}$
}

\section{Resumen}

El estudio de la biomasa íctica de las lagunas costeras fue realizado en las lagunas costeras de Bihmuna, Pahara, Wounhta y Karatá. Tuvo como propósito determinar la biomasa presente de los recursos pesqueros existente en las cuatro lagunas estudiadas en la época de verano e invierno y abordar la problemática actual que existe en estas lagunas para la regulación pesquera.

Este estudio de corte cuantitativo utilizó cuatro unidades de muestreos (atarraya, redes agalleras, red de cerco y aro jaibero) y de manera aleatoria al azar fueron seleccionado los puntos de muestreos en las lagunas; los cuales fueron por un periodo de tiempo de 24 horas (diurno y nocturno), donde se utilizaron diferentes aperos de pesca. En relación a los resultados, la biomasa de las lagunas fue mayor en invierno; mientras que, en el verano hubo mayor presencia de individuos en estados juveniles y pre juveniles.

En el muestreo de verano hubo captura de peneidos en la laguna de Bihmuna y Karatá donde los individuos capturados en su mayor parte eran de estado de madurez pre juvenil. Es importante mencionar que la laguna de Bihmuna en la época de invierno hubo presencia de peneidos; esto se debe por la circulación de sales disueltas en el agua que es producto de la entrada de agua del océano que ingresa en esta laguna a través de las dos desembocaduras que presenta.

Palabras clave: Biomasa; peneidos; recursos pesqueros; desembocadura.

\section{Abstract}

The study of the fish biomass of the coastal lagoons was carried out in the coastal lagoons of Bihmuna, Pahara, Wounhta and Karatá. Its purpose was to determine the present biomass of the fishing resources existing in the four lagoons studied during the summer and winter seasons and to address the current problems that exist in these lagoons for fisheries regulation.

This quantitative approach study used four sampling units (atarraya, gillnets, purse seine net and ring) and random sampling points were selected in the lagoons; which were for a period of 24 hours (day and night), where different fishing tools were used. In relation to the results, the biomass of the lagoons was higher in winter; however in the summer there was a greater presence of individuals in juvenile and pre-juvenile states.

\footnotetext{
Licenciado en Biología marina, Técnico de IREMADES, URACCAN, No. ORCID: http://orcid.org/0000-0002-1931-577X ; e-mail; ronaldw1981@yahoo.com

2 Msc. Biólogo Marino con especialidad en prevención y mitigación de desastres naturales, e-mail: dchavarria84@yahoo.com

3 Msc. en Gobernanza y Desarrollo Con Identidad, Director de IREMADES-CISA, URACCAN, e-mail: marcoswillni123@gmail.com ORCID: : http://orcid.org/00000002-1887-1328
}

Recibido: 16/08/2019 - Aprobado: 30/08/2019 
In the summer sampling, there were catches of penaeids in the lagoon of Bihmuna and Karatá where the individuals captured for the most part were of a state of pre-juvenile maturity. It is important to mention that the Bihmuna lagoon in the winter time there was the presence of penaeids; this is due to the circulation of dissolved salts in the water that is the product of the entry of water from the ocean that enters this lagoon through the two mouths it has.

Keywords: Biomass; penaeids; fishing resources; river mouth

\section{Introducción}

En el marco del proyecto "Fortalecimiento de condiciones y capacidades de la población para la conservación y manejo sostenible de los ecosistemas costeros de la Costa Caribe de Nicaragua/ ENV/380/524", fue realizado el estudio de la biomasa de cuatro lagunas más importantes del Caribe Norte de Nicaragua como parte de los estudios complementarios de la actualización del plan de manejo de la reserva de los Cayos Miskitos.

Los muestreos fueron llevados a cabo en verano como en invierno, con el fin de determinar la dinámica poblacional de las especies existentes en las lagunas de Bihmuna, Pahara, Karatá y Wounhta. Se fundamentó en los siguientes criterios básicos: mediciones biomorfológicas, peso de los individuos capturados, estado de madurez sexual, sexo, número de lances, calado, peso total por especie, mediciones de los parámetros físicos químicos, presencia de fitoplancton y zooplancton. La variable seleccionada sirve para determinar la biomasa de las lagunas costeras y evaluar el estado de salud en que se encuentra dichas lagunas.

Este estudio, servirá de base para integrar en la actualización del plan de manejo de la reserva de los Cayos Miskitos, regulaciones pesqueras, acorde con la realidad de los recursos pesqueros existentes en las lagunas que será de utilidad para un aprovechamiento sostenible y sustentable de los recursos pesqueros existentes y que las futuras generaciones puedan percibir de los beneficios derivados de la pesquería existente en dichas lagunas.

\section{Revisión de la literatura}

\section{Importancia de los ecosistemas costeros-marinos}

En el plan ambiental de Nicaragua 2000-2005, existe una lista de sitios que podrían considerarse de patrimonio universal y dentro de esta lista aparece la reserva Biológica de los Cayos Miskitos en el Caribe y actualmente el comité internacional del programa sobre el hombre y la biósfera de UNESCO ha reconocido a los Cayos Miskitos como reserva de biósfera (PANIC, 2006).

Dentro del área de la reserva de los Cayos Miskitos existe una diversidad de ambientes costeros-marinos que interactúan entre sí, comprendiendo una de las mayores extensiones de pastos marinos en el caribe, entremezclados con arrecifes de coral. Además, dentro de la reserva existe una diversidad de ambientes costeros pocos intervenidos por el hombre como son los bosques de manglares, humedales y turberas; lo que ubica entre las áreas costeras- marina biológicamente más ricos en América tropical (Marena, 2002).

Una de las características naturales más importantes de la Reserva, es la presencia de gran cantidad de lagunas costeras, en la cual, la mayoría de estas lagunas son de poca profundidad (4 a $5 \mathrm{~m}$ ) y están conectadas con tierra firme por multitud de ríos, lagunas de agua dulce y pantanos salobres. Este complejo de ambientes estuarinos de la reserva, se extiende por centenares de kilómetros de longitud, desde Cabo Gracias a Dios hasta Prinzapolka.

La mayoría de los productos comerciales llegan a Bilwi desde las comunidades miskitas por embarcaciones que navegan cruzando las lagunas costeras y las aguas costeras oceánicas. Además, estas lagunas 
costeras son hábitats sumamente importantes desde el punto de vista de conservación de la biodiversidad y el mantenimiento de las economías locales. Su papel en el mantenimiento del ciclo de vida de los organismos costero-marinos incluyendo las especies para pesca comercial es invaluable.

La alta diversidad de ecosistemas costeros que forma la reserva de los Cayos Miskitos y su franja costera ha permitido a las especies en zonas de alimentación, reproducción y crianza, desove y reclutamiento conformando ecosistemas para la sobrevivencia de tortugas verdes, camarones, langostas, manatíes, aves acuáticas, delfines, peces, corales. Sin mencionar la riqueza genética invalorable que conforman en las lagunas costeras.

La laguna de Bihmuna se encuentra ubicada en el norte de Puerto Cabezas y tiene una superficie de $131.68 \mathrm{~km}$ y en cuya laguna se encuentra el poblado de Bihmuna. La laguna Pahara ubica en el norte de Puerto Cabeza y colinda con la laguna de Krukira, tiene una superficie de $91.97 \mathrm{~km}$ y en esta laguna se ubica la comunidad de Pahara y esta laguna es compartido entre las comunidades de Pahara y Awastara, en lo que respecta a la laguna de Karatá se encuentra ubicado en el sur de Puerto Cabezas en cuya laguna asienta la comunidad de Karatá y tiene una extensión de $29.75 \mathrm{~km}$ y la Laguna de Wounhta, conocida también en la zona como "Laguna de Halover" por los lugareños, se encuentra ubicada en el litoral Sur del municipio de Puerto Cabezas. En sus costas están la Comunidad de Halover y la Comunidad de Wouhnta, tiene una superficie de $93.83 \mathrm{~km}$.

\section{Evaluación de la salud de las lagunas naturales en la Reserva Biológica Cayos Miskitos}

FUNDAR (2018), afirma que las lagunas naturales de la reserva de los Cayos Miskitos presentan una buena condición de salud. Esto principalmente porque se mantiene las condiciones ambientales y naturales de los ecosistemas que las rodean. Además, que no existen fábricas ni industrias que aporten descargas de contaminantes químicos, ni grandes descargas de desechos domiciliares. Pero, en cuanto a los valores de sílice en la laguna Bihmuna y Wouhnta presenta valores de 7,56 $\mathrm{mg}^{-\mathrm{L}^{-1}}$ que significa que en la parte alta de la laguna están utilizando químicos y que estos son traídos por los ríos que desembocan en estas lagunas.

\section{Materiales y métodos}

Esta investigación fue de tipo cuantitativo, efectuada durante las estaciones de verano e invierno con muestreos continuos de 24 horas en las lagunas de Bihmuna, Pahara, Karatá y Wounhta en donde los puntos de muestreos fueron seleccionados de manera aleatoria estratificada; en la cual, realizo muestreos en diferentes puntos de las lagunas: orilla, centro, afluentes de los ríos y desembocadura de la laguna con el océano.

\section{Métodos de muestreos}

Para los muestreos, se utilizó una atarraya de 6 pies de largo y 10 pies de diámetro con luz de malla de $1 / 4$ de pulgada, operada por una persona desde una embarcación tipo panga se realizó lances de atarraya. Además, en varios puntos de las lagunas fueron colocadas redes agalleras de 4 y 5 pulgadas de luz de malla. Pero, este arte de pesca fue utilizado solamente en la noche con el fin de capturar peces nocturnos de mayor tamaño y comercialmente importante; para ello, los lances tuvieron una duración de 4 horas en donde fueron revisados y caladas. Además, se utilizó red de cerco en donde realizo varios arrastres en los diferentes puntos de las lagunas este con el fin de capturar peces pelágicos, epipelágicos y demersales.

Otro de los aperos de pesca utilizada en el muestreo de la biomasa de las lagunas fue la trampa para Jaiba conocido como aro jaibero, en la cual colocada la carnada en las trampas y calado cada 15 minutos. Este apero de pesca fue utilizado para determinar la existencia de la población de jaibas en las lagunas. Asimismo, los individuos capturados fueron pesados con una balanza digital en donde se obtuvo los datos 
de la biomasa por especies y por arte de pesca de igual manera con un ictiometro se obtuvo información como: longitud total, longitud estándar, longitud circunferencial, longitud horquilla, estado de madurez sexual, número de lances, número de redes y sexo.

\section{Muestreo de la calidad de agua de las lagunas}

Para obtener el dato de la profundidad, se utilizó plomo y una cuerda graduada en centímetro y por sitios de muestreos fueron medidas estos datos y posteriormente fue calculada la profundidad promedio por lagunas.

Además, se obtuvo información de los parámetros físicos químicos de agua de lagunas como; la temperatura, $\mathrm{Ph}$, conductividad eléctrica, salinidad y turbidez; para ello, fue utilizado un phmetro- conductivímetro portátil, salinómetro de mercurio y el turbímetro. Estos parámetros, fueron analizados tanto en verano como invierno.

\section{Procesamientos de datos}

Para el cálculo de la biomasa de la muestra poblacional utilizó la siguiente formula;

$$
\begin{array}{ll}
\mathrm{B}=\mathrm{P} / \mathrm{A} ; & \text { Donde} ; \mathrm{B}=\text { Biomasa en } \mathrm{gr} / \mathrm{m}^{2} \\
& \mathrm{P}=\text { Peso total } \\
\mathrm{A}=\text { área muestreada }
\end{array}
$$

Fórmula utilizada para el cálculo de la biodiversidad de la cuenca del rio Orinoco, Venezuela, estudio realizado por; pablo Ramírez Villarreal investigador y profesor de la universidad de oriente; instituto de ciencias del mar y limnología.

En cuanto, a la fórmula utilizada para el cálculo de la biomasa total por lagunas fue utilizado la siguiente: fórmula: $\mathrm{B}=$ Biomasa de la muestra calculado $\mathrm{x}$ área total de la laguna.

\section{Resultados y discusión}

En el estudio de la biomasa de las lagunas costeras, en el invierno presentan mayor biomasa ya que hubo más captura y los especímenes eran de talla juvenil y adultos y la laguna con mayor biomasa presentada fue Bihmuna, seguida de la laguna de Wounhta, mientras que la de Pahara presenta la biomasa más baja. En cuanto, a la estación de verano la laguna que presentó mayor biomasa fue Wounhta donde predomina las medusas y la laguna de Karatá fue la segunda laguna con mayor biomasa calculada.

En relación, a la biomasa poblacional de las lagunas costeras, Bihmuna en el invierno presenta mayor biomasa, seguida de la laguna de Wounhta, mientras que la laguna de Karatá muestra biomasa menor; esto debido, a la superficie de esta laguna que es la más pequeña de las tres.

En tanto, que, en el verano, la laguna de Wounhta muestra mayor biomasa poblacional, posteriormente la laguna de Bihmuna, mientras que la laguna de Karatá presentó menor biomasa. Pero, como se mencionaba anteriormente, esto debido a su superficie ya que en relación a la diversidad y abundancia de especies esta laguna está mejor que la laguna de Pahra. Las capturas de peneidos (camarones), solamente fue en las lagunas de Bihmuna y Karatá en la época de verano como indica el cuadro No. 2 donde la mayoría de los especímenes eran de estado de madurez pre juvenil. 


\section{Biomasa de peneidos en las lagunas naturales}

Es importante mencionar, la presencia de peneidos en la época lluviosa en la laguna de Bihmuna, esto se debe que esta laguna presentó salinidad en el agua de 10 ppm (ver grafica 2); por ello, existe la presencia de esta especie que habitan en aguas salobres con salinidades que varían de 10- $25 \mathrm{ppm}$ de sal.

Asimismo, en el verano a pesar que las lagunas de Pahara y Wounhta presentaron salinidades que estaban entre 12 y 13 ppm; en estas lagunas no hubo presencia de camarones. Además, la biomasa presentada fue lo más bajo en comparación con las biomasas de escamas y medusas en el verano en la cual, la laguna de Bihmuna mostró mayor biomasa de esta especie de 1.49 gr por metro cuadrado.

Cuadro No. 2. Biomasa de camarones en las lagunas en el verano e invierno

\begin{tabular}{|l|l|l|l|l|l|l|l|}
\hline \multirow{2}{*}{ Laguna } & \multirow{2}{*}{$\begin{array}{c}\text { Área de mues- } \\
\text { treo }\left(\mathbf{m}^{2}\right)\end{array}$} & \multicolumn{2}{c|}{ Peso Total Capturado $(\mathbf{g r})$} & \multicolumn{2}{c|}{ Biomasa por $\mathbf{m}^{2}(\mathbf{g r})$} & \multicolumn{2}{c|}{ Biomasa Total $(\mathrm{Kg})$} \\
\cline { 3 - 9 } & & Verano & \multicolumn{1}{c|}{ Invierno } & Verano & Invierno & \multicolumn{1}{c|}{ Verano } & Invierno \\
\hline Bihmuna & 2700 & 4036 & 1150 & 1.49 & 0.43 & 196,203 & 56,622 \\
\hline Pahara & 2650 & 0 & 0 & 0 & 0 & 0 & 0 \\
\hline Karatá & 1560 & 1122 & 0 & 0.73 & 0 & 21,718 & 0 \\
\hline Wounhta & 2600 & 0 & 0 & 0 & 0 & 0 & 0 \\
\hline
\end{tabular}

\section{Biomasa de escamas en las lagunas naturales}

Lo que respecta a los escamas (peces), en el estudio de la biomasa de las lagunas costeras, en el verano la mayoría de los individuos capturados fueron juveniles y pre juveniles de las siguientes especies: palometas, sardinas, róbalo, mojarra, pargo de estero, lisa, guapote, roncador, lenguado y jurel; mientras que, en el invierno las capturas en su mayoría fueron individuos juveniles y algunos ejemplares adultos de las especies: barbudo o tunki, pis pis, palometas, mugil curema, roncador, sardinas, róbalo, pez aguja, pargo lagunar, jurel, macarela, pez hoja, mojarra, pez globo, bagre marino y sábalo real (Megalops atlanticus) de las cuales, la laguna de Bihmuna la mayoría de las especies capturados fueron de la especie Caranx.

La laguna de Bihmuna presentó 12 escamas, en la cual la especie Caranx (Jurel) fue de mayor captura con 38 individuos, con un peso de $27.84 \mathrm{~kg}$. En cuanto a la especie centropomus (róbalos) muestra una captura de $4.72 \mathrm{Kg}$ equivalente a 12 individuos y en esta laguna en el invierno capturó 15 individuos de sardinas que hace una laguna con presencia de peces mayores carnívoros como los especies Caranx.

Es importante recalcar, que la laguna de Karatá es donde hubo más captura de la especie Centropomus (róbalos), especie de mayor valor comercial en el mercado regional y nacional. En cambio, la laguna de Wounhta fue la laguna que presentó mayor diversidad de especies de escamas con 14 especies. Mientras que la laguna de Pahara fue la laguna que presentó diversidad y riqueza baja en relación a las otras lagunas naturales.

Cuadro No. 3. Biomasa de peces en las lagunas en el verano e invierno

\begin{tabular}{|l|l|l|l|l|l|l|l|}
\hline \multicolumn{1}{|c|}{ Laguna } & $\begin{array}{c}\text { Área de mues- } \\
\text { treo }\left(\mathbf{m}^{2}\right)\end{array}$ & \multicolumn{2}{|c|}{$\begin{array}{c}\text { Peso Total capturado } \\
(\mathrm{Gr})\end{array}$} & \multicolumn{2}{c|}{ Biomasa por $\mathbf{m}^{2}(\mathrm{Gr})$} & \multicolumn{2}{c|}{ Biomasa Total (kg)) } \\
\hline & & Verano & Invierno & Verano & Invierno & \multicolumn{1}{|c|}{ Verano } & \multicolumn{1}{|c|}{ Invierno } \\
\hline Bihmuna & 2700 & 8475 & 42400 & 3.85 & 15.7 & 506,968 & $2,067,376$ \\
\hline Pahara & 2650 & 2014 & 14450 & 0.76 & 5.5 & 69,897 & 505,835 \\
\hline Karatá & 1560 & 6009 & 18800 & 2.41 & 10.7 & 71,697 & 359,975 \\
\hline Wounhta & 2600 & 6274 & 27930 & 3.5 & 12.1 & 328,405 & $1,135,343$ \\
\hline
\end{tabular}


En las lagunas costeras en el verano, existen una población abundante de cardúmenes de sardinas donde la laguna de Bihmuna, presenta mayor número de individuos que hace una laguna con alta diversidad íctica y donde la mayoría de los individuos son de estado juveniles; mientras, la laguna de Pahara es una laguna con baja diversidad y abundancia ya que durante el verano solamente fueron capturadas 3 especies de escamas con biomasa de 0.76 gr por metro cuadrado.

La laguna de Wounhta fue la laguna con mayor abundancia íctica donde hubo dominancia de dos especies (sardinas y palometas). En cambio, la laguna de Karatá presenta mayor diversidad y riqueza íctica. Pero, baja abundancia de los individuos de las especies encontradas.

\section{Biomasa de medusas en las lagunas naturales}

En cuanto a las medusas en el verano, en las lagunas naturales, hubo dominancia y abundancia de esta especie en relación a las otras especies capturadas donde la mayor presencia de esta especie fue en las lagunas de Wounhta y Pahara.

Cuadro No. 4. Biomasa de medusas en las lagunas en el verano e invierno

\begin{tabular}{|l|l|l|l|l|l|l|l|}
\hline \multirow{2}{*}{ Laguna } & \multirow{2}{*}{$\begin{array}{c}\text { Área de mues- } \\
\text { treo }\left(\mathbf{m}^{2}\right)\end{array}$} & \multicolumn{2}{|c|}{ Peso Total $(\mathbf{g r})$} & \multicolumn{2}{c|}{ Biomasa por $\mathbf{m}^{2}(\mathbf{g r})$} & \multicolumn{2}{c|}{ Biomasa Total $(\mathbf{k g}))$} \\
\cline { 3 - 9 } & & Verano & Invierno & Verano & Invierno & Verano & Invierno \\
\hline Bihmuna & 2700 & 5731 & 942 & 2.16 & 1.35 & 198,655 & 177,768 \\
\hline Pahara & 2650 & 7760 & 0 & 2.95 & 0 & 377,921 & 0 \\
\hline Karata & 1560 & 881 & 0 & 0.86 & 0 & 25,585 & 0 \\
\hline Wounhta & 2600 & 13331 & 0 & 5.13 & 0 & 481,348 & 0 \\
\hline
\end{tabular}

En el invierno, esta especie solamente se encontró en la laguna de Bihmuna debido que es una laguna salobre, hábitat para estas especies, por ello, no fue encontrado en las lagunas de karatá, Pahara y Wounhta donde son completamente de agua dulce. Como muestra, en el cuadro anterior en el verano, la laguna Wounhta, fue la que presentó mayor biomasa de 5.13 gr por metro cuadrado; mientras, la laguna de Karatá presentó biomasa menor de medusas de o.86 gr por metro cuadrado.

\section{Biomasa de Callinectus en las lagunas costeras}

Las poblaciones de jaibas en las cuatro lagunas muestreadas se encuentran bien representadas y es un indicador del buen estado de salud de la laguna y es en la época seca donde existe mayor población de esta especie, ya que es una especie salina y durante el invierno las capturas fueron mayores de machos juveniles y hembras inmaduras; mientras que, en el verano, se presentan más hembras maduras en muda y machos adultos. Cabe mencionar, que estos individuos fueron capturados en mayor cantidad en la estación de verano donde las lagunas alcanzan salinidades de hasta $20 \mathrm{ppm}$.

Cuadro No. 5. Resultados de la Biomasa de Callinectus en las lagunas de RACCN

\begin{tabular}{|c|c|c|c|c|c|c|c|c|}
\hline \multirow[t]{2}{*}{ Lagunas } & \multirow{2}{*}{$\begin{array}{l}\text { Superficie } \\
\text { Total (km) }\end{array}$} & \multirow{2}{*}{$\begin{array}{l}\text { Sitios muestrea- } \\
\text { dos }\end{array}$} & \multicolumn{2}{|c|}{$\begin{array}{c}\text { Captura por laguna } \\
\text { (gr) }\end{array}$} & \multicolumn{2}{|c|}{ Biomasa por $\mathrm{m}^{2}$ (gr) } & \multicolumn{2}{|c|}{ Biomasa Total (Kg) } \\
\hline & & & Invierno & verano & Invierno & verano & Invierno & Verano \\
\hline Bihmuna & 131.68 & 18 & 4370 & 5376 & 1.62 & 2.21 & 213,322 & 291,013 \\
\hline Pahra & 91.97 & 18 & 2360 & 2998 & 0.62 & 1.13 & 57,021 & 103,640 \\
\hline Karatá & 29.75 & 18 & 2845 & 3270 & 1.52 & 1.87 & 45,220 & 55,741 \\
\hline Wounhta & 93.83 & 18 & 3896 & 4618 & 1.58 & 1.77 & 148,251 & 166,397 \\
\hline
\end{tabular}


Para el muestreo de jaiba (Callinectus), en las lagunas costeras fue realizado con 16 unidades de aro jaibero, en la cual, en el verano las biomasas de esta especie fueron mayor donde la laguna de Bihmuna presentó mayor biomasa de esta especie de 2.21 gr por metro cuadrado mientras que la laguna de Pahara presento la biomasa menor de 1.13 gr por metro cuadrado. Asimismo, en el invierno, tanto la laguna de Bihmuna y Pahara fueron los que presentan mayor y menor biomasa de Callinectus.

\section{Resultados de los parámetros físico químicos de las lagunas naturales}

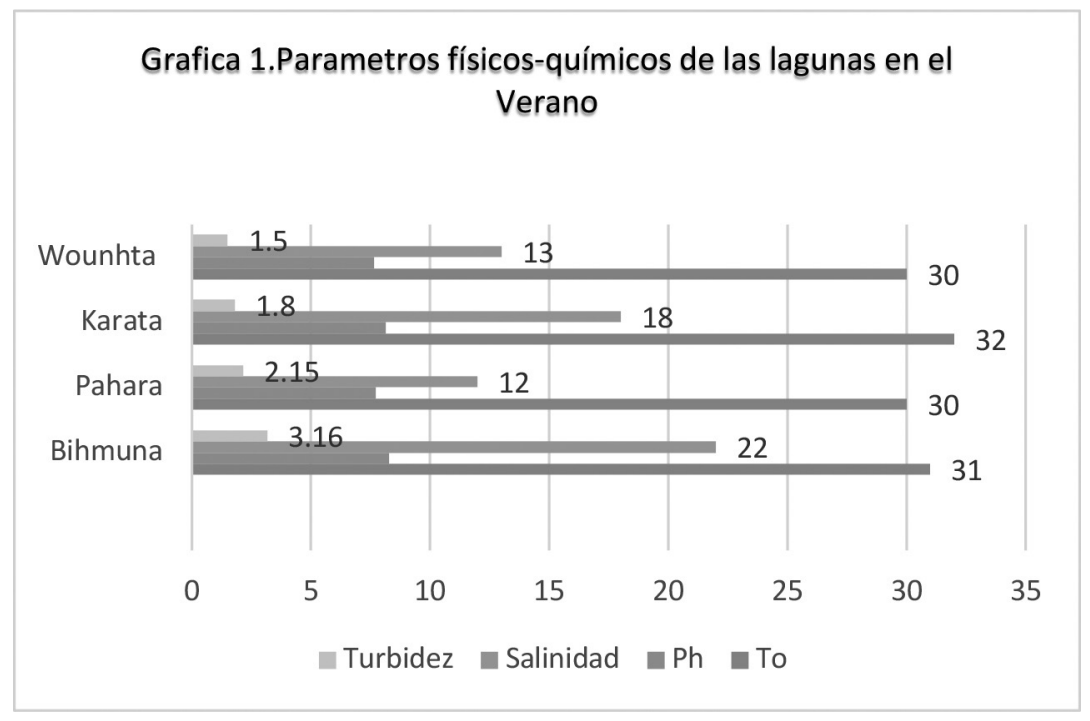

El análisis de las muestras de agua de las cuatro lagunas naturales, indican un ambiente alcalino con valores superiores a 7, donde la laguna de Bihmuna, presentó el valor de $\mathrm{pH}$ más alto con 8.28, mientras que la laguna de Wounhta presento el valor más bajo con 7.64. En cuanto, al valor de la temperatura de agua en las lagunas en verano e invierno no difieren significativamente, mientras que los valores de salinidad y turbidez difieren significativamente presentando en el verano la laguna de Bihmuna con mayor salinidad (22 ppm), mientras que la laguna de Pahara muestra menor salinidad de $12 \mathrm{ppm}$; esta diferencia en los valores de la salinidad es debido que la laguna de Bihmuna presenta dos salidas al mar (desembocadura) donde entra mayor cantidad de agua de océano, mientras que la laguna de Pahara tiene una distancia considerable de la barra; por ende, la circulación de sales en el agua es menor.

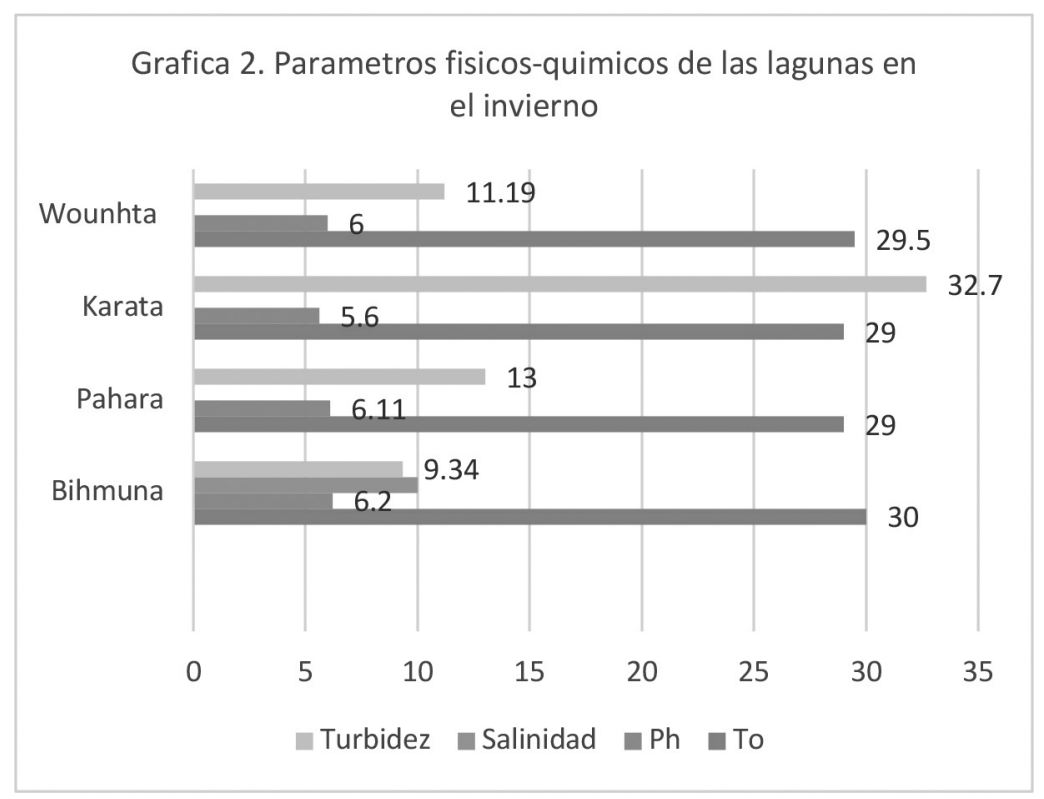


En relación, a la turbidez en el invierno las lagunas naturales presentan alta turbidez debido a la descarga de los ríos que alimentan a estas lagunas donde la laguna de Karatá presentó mayor turbidez de 32.7 NTU, mientras que en el verano existe baja turbidez donde laguna de Wounhta presentó menor valor de 1.5 NTU.

\section{Conclusiones}

En el estudio de la biomasa de las lagunas costeras en el invierno las capturas de escamas fueron mayores en comparación con el verano donde hubo una cantidad considerable de individuos adultos. En cuanto a los camarones solamente se captura en la laguna de Bihmuna donde en su mayoría eran de estado de madurez pre juvenil. Es importante mencionar que la presencia de camarones en la época lluviosa en la laguna de Bihmuna se debe que esta laguna presenta sales; por ello, existe la presencia de esta especie.

En cuanto, al estado de madurez de las escamas capturados en los sitios muestreados en la época seca en su mayoría fueron juveniles y pre juveniles de las siguientes escamas; palometas, sardinas, robalo, mojarra, pargo de estero, lisa, guapote, roncador, lenguado y jurel, mientras que en el invierno capturo más individuos adultos y juveniles de las siguientes especies; Robalo, roncador y sábalo real (Megalops Atlanticus), solamente los individuos de camarones capturados eran de fase pre juveniles y juveniles.

Asimismo, lo que respecta a la biomasa de camarones en el verano en las lagunas naturales el resultado fue bajo, obteniéndose datos sólo en las lagunas de Bihmuna y Karatá. En las lagunas de Pahara y Wouhnta no se lograron capturas de esta especie. Es importante mencionar que la laguna de Bihmuna, presenta mayor biomasa de esta especie de $1.49 \mathrm{gr}$ por metro cuadrado, esto lo que respecta en el verano.

Asimismo, en las cuatro lagunas muestreadas durante el verano presenta una gran cantidad de medusas donde la mayor presencia de esta especie fue en la laguna de Wounhta (grafica 1), seguido de la laguna de Pahra mientras que en la época lluviosa esta especie no se capturo ya que son especies salinas que habitan en salinidades mayores de $10 \mathrm{ppm}$. En las cuatro lagunas muestreadas durante el verano presenta una gran cantidad de medusas donde la mayor presencia de esta especie fue en la laguna de Wounhta (grafica 1), seguido de la laguna de Pahra mientras que en la época lluviosa esta especie no se capturo ya que son especies salinas que habitan en salinidades mayores de $10 \mathrm{ppm}$.

En cuanto a la profundidad promedio de estas lagunas no difiere mucho entre sí, la laguna de Pahara es el que presenta mayor profundidad; mientras que la de Bihmuna presenta menor profundidad. La profundidad y la transparencia del agua no difieren significativamente entre las cuatro áreas de estudio.

La laguna de Bihmuna presenta menor transparencia, mientras la laguna de Wounhta presenta mayor transparencia del agua. Asimismo, se capturó un mayor número de individuos adultos de escamas en la laguna de Wounhta, mientras que en los otros fueron más individuos en su estado pre juvenil y juvenil.

Las poblaciones de jaibas en las cuatro lagunas muestreadas se encuentran bien representados y es un indicador del buen estado de salud de la laguna y es en la época seca donde existe mayor población de esta especie ya que es una especie salina y durante el invierno las capturas fueron mayores de machos juveniles y hembras inmaduras, mientras que en el verano presenta más hembras maduras en muda y machos adultos. Estos individuos fueron capturados en mayor cantidad en la época seca donde las aguas de estas lagunas alcanzan salinidades de hasta $20 \mathrm{ppm}$.

El análisis de las muestras de agua tomadas en las cuatro lagunas naturales, indican un ambiente alcalino con valores superiores a 7. La laguna Bihmuna, presento el valor de $\mathrm{pH}$ más alto con 8.28 , mientras que la laguna de Wounhta presento el valor más bajo con 7.64.

\section{Agradecimiento}

Esta publicación obtuvo el financiamiento del Proyecto Ecosistemas Costeros, auspiciado por: Unión Europea, Dka Austria, Cooperación Austriaca para el Desarrollo y Horizontzooo. 


\section{Lista de referencias}

Arauz J. C. (2001). Caracterización de las especies de peces de valor comercial de la Bahía de Bluefields y Sus Alrededores. Bluefields, Nicaragua, octubre, 2001. 45 pág.

Castro L, J. (1999). Ictiofauna de Estuarino Lagunar y Vicarria de México, Baja California. 1er Edición, Editorial Limusa, Pag 705.

Cotto A. (2001). Guía de Identificación de Peces Marinos del Mar Caribe de Nicaragua. Bluefields. Nicaragua. DIPAL II. 82 pag

Gregory, S.Gilberth, (2002). Manual para las Investigaciones de Biología de Campo. Instituto Smithsonia de Investigaciones Tropicales, Universidad de Panamá, 2da. Edición. Pag 130.

FAO (1978). Identificación de peces del Atlántico Central oeste. Pág. 34,

Karlen, L. Bjarnason, (1989). La Pesca Artesanal con redes de Enmalle de deriva. FAO Documento Técnico de Pesca, Universidad de Tromson Noruega, pág. 60.

M. Ramón, B. Tim, A. Bertha (1985). Estudio sobre la calidad y utilización de algunas especies de peces dermasal de tamaño pequeño en aspecto de Comportamiento y Biología, Guayaquil, Ecuador. 33 Pág.

Pérez, M. (2001). Biología pesquera del Bagre (Bagre marinus), en Lagunas Costera de la Región Autónoma Atlántico Sur, Nicaragua. DIPAL II, Laguna de perlas, Nicaragua. 53 Pág.

Pérez, M. (2001). Biología pesquera de la lisa (Mugil curema), en Lagunas Costera de la Región Autónoma Atlántico Sur, Nicaragua. DIPAL II, Laguna de Perlas, Nicaragua. 53 Pág.

Sánchez, R. (2001). Biología eesquera del róbalo (Género Centropomus), en lagunas Costera de la Región Autónoma Atlántico Sur. Nicaragua, DIPAL II, Laguna de Perlas, Nicaragua, Pág. 105.

Zúñiga H. E. (1981). Manual de Campo de los Peces más Comunes del Atlántico de Nicaragua. Pág. 56. 